\title{
Human Adenovirus Type 55 Distribution, Regional Persistence, and Genetic Variability
}

\author{
Jun Hang, Adriana E. Kajon, Paul C.F. Graf, ${ }^{1}$ Irina Maljkovic Berry, Yu Yang, Mark A. Sanborn, \\ Christian K. Fung, Anima Adhikari, Melinda S. Balansay-Ames, Christopher A. Myers, \\ Leonard N. Binn, Richard G. Jarman, Robert A. Kuschner, Natalie D. Collins
}

\begin{abstract}
Human adenovirus type 55 (HAdV-55) causes acute respiratory disease of variable severity and has become an emergent threat in both civilian and military populations. HAdV-55 infection is endemic to China and South Korea, but data from other regions and time periods are needed for comprehensive assessment of HAdV-55 prevalence from a global perspective. In this study, we subjected HAdV-55 isolates from various countries collected during 1969-2018 to whole-genome sequencing, genomic and proteomic comparison, and phylogenetic analyses. The results show worldwide distribution of HAdV-55; recent strains share a high degree of genomic homogeneity. Distinct strains circulated regionally for several years, suggesting persistent local transmission. Several cases of sporadic introduction of certain strains to other countries were documented. Among the identified amino acid mutations distinguishing HAdV-55 strains, some have potential impact on essential viral functions and may affect infectivity and transmission.
\end{abstract}

\begin{abstract}
A cute respiratory diseases (ARD) are caused by numerous viral pathogens, including several human adenovirus (HAdV) types. Respiratory HAdV infections range from mild to severe and are fatal in some cases. HAdV-associated respiratory disease has threatened military readiness, and an increasing number of outbreaks and isolated cases documented in civilian communities in the United States and other countries (1-4) indicate that it is an emerging threat to public health. The live oral HAdV type 4 and type 7 vaccines are highly effective against the 2 dominant

Author affiliations: Walter Reed Army Institute of Research, Silver Spring, Maryland, USA (J. Hang, I.M. Berry, Y. Yang. M.A. Sanborn, C.K. Fung, A. Adhikari, L.N. Binn, R.G. Jarman, R.A. Kuschner, N.D. Collins); Lovelace Respiratory Research Institute, Albuquerque, New Mexico, USA (A.E. Kajon); US Naval Health Research Center, San Diego, California, USA (P.C.F. Graf, M.S. Balansay-Ames, C.A. Myers)
\end{abstract}

DOI: https://doi.org/10.3201/eid2607.191707 types causing ARD outbreaks in the military environment, but they elicit type-specific immunity and are restricted to use in the US military (5-7). ARD caused by other HAdV types, such as HAdV types 3, 14, and 21 , occurs sporadically in military basic training facilities (8-10). An increasing number of new HAdV types have been described, indicating the potential emergence of new HAdV-associated diseases (11-13).

HAdV-55 was initially identified in respiratory isolates originating in China during 1965-1981 as serotype $11 \mathrm{a}$, a distinct genomic variant of serotype 11, by its distinct BamHI digestion profile compared with the HAdV-11 prototype strain Slobitski (14). This unique subspecies HAdV-B2 genotype was designated HAdV-55 (P14H11F14) in 2013 after the bioinformatics analysis of complete genome sequences revealed that the genome consists of a HAdV-14 backbone with a portion of the hexon gene from HAdV-11 (15-17). HAdV-55 has reemerged as a prevalent ARD pathogen, with endemic circulation reported in China and South Korea since 2006 (18-21). HAdV-55 whole-genome sequences (WGS) available in GenBank are mainly representative of strains from China and South Korea, but also include strains from Argentina, Egypt, and Singapore (Table 1) (22-25). More data for HAdV-55 isolates from other global locations and years are needed to enable a comprehensive investigation of the spectrum of intratypic genetic variability, phylogeny, and evolution of HAdV-55, and to apply the knowledge to epidemiology and preventive measures. We acquired WGSs of $72 \mathrm{HAdV}-55$ clinical isolates from 1969-2018 in 6 countries, and conducted genomic, proteomic, and phylogenetic analyses to reveal unique characteristics of HAdV-55 and identify amino acid residue differences between strains.

${ }^{1}$ Current affiliation: US Naval Medical Research Unit 6, Lima, Peru. 
Table 1. HAdV type 55 strains from other studies used in genomic analysis for study of virus distribution, regional persistence, and genetic variability

\begin{tabular}{|c|c|c|c|c|}
\hline HAdV-55 strain & Collection year & Collection date & Country & $\begin{array}{c}\text { GenBank } \\
\text { accession no. }\end{array}$ \\
\hline Human/EGY/ak37_AdV11a/2001/55[P14H11F14] & 2001 & May 1 & Egypt & JX423385 \\
\hline Human/ARG/ak36_AdV11a/2005/55[P14H11F14] & 2005 & Jan 1 & Argentina & JX423384 \\
\hline SGN1222 - & 2005 & May 26 & Singapore & FJ597732 \\
\hline QS-DLL & 2006 & Apr & China & FJ643676 \\
\hline CQ-814 & 2010 & Aug 18 & China & JX123027 \\
\hline HAdV-B/CHN/BJ01/2011/55[P14H11F14] & 2011 & Mar 24 & China & JX491639 \\
\hline Shanxi/QZ01/2011 & & Dec 5 & China & KJ883522 \\
\hline CQ-1657 & & Apr 22 & China & JX123028 \\
\hline Shanxi-Y16 & & UNK & China & MK123979 \\
\hline Human/CHN/AQ-1/2012/55[P14/H11/F14] & 2012 & Apr 18 & China & KP279748 \\
\hline HAdV-B55 XZ2012-492 & & Apr 26 & China & KC857701 \\
\hline CQ-2903 & & Jan 8 & China & JX123029 \\
\hline Hebei/BD01/2012 & & Feb 11 & China & KP896478 \\
\hline Hebei/BD6728/2013 & 2013 & Apr 6 & China & KJ883520 \\
\hline TJ-2013-90 & & Jan 14 & China & KF908851 \\
\hline TY12 & & UNK & China & MK123980 \\
\hline TY26 & & UNK & China & MK123981 \\
\hline Liaoning/LS01/2013 & & Feb 25 & China & KP896483 \\
\hline Tianjin/TJ01/2013 & & Jan 15 & China & KP896484 \\
\hline Hebei/BD6729/2013 & & Apr 7 & China & KJ883521 \\
\hline JS201501 & 2015 & Nov 12 & China & KX289874 \\
\hline 100-GD_CHN_2016 & 2016 & Jun & China & KY780931 \\
\hline 73-GD_C̄HN_2016 & & Jun & China & KY780933 \\
\hline 60-GD-2016 & & Jun 30 & China & KY070248 \\
\hline Yunnan/KM04/2016 & & Jun 8 & China & KY002685 \\
\hline AFMC 16-0011 & 2016 & Feb 23 & South Korea & KX494979 \\
\hline 267 & 2018 & Jun & China (Guangzhou) & MK123978 \\
\hline
\end{tabular}

\section{Materials and Methods}

HAdV-55 Strains, DNA Extraction, Genome Sequencing The HAdV-55 isolates included in this study were acquired from 2 main sources: the archival collection of isolates at Lovelace Respiratory Research Institute (LRRI), Albuquerque, NM, USA, gathered through collaborative surveillance efforts funded by the US Department of Defense's Global Emerging Infections Surveillance and Response System; and the HAdV-positive specimen collection of the Naval Health Research Center-Operational Infectious Disease (NHRC-OID, San Diego, CA, USA), NHRC-OID conducts surveillance of febrile respiratory illness among military personnel and their dependents in the Pacific Rim at Commander US Fleet Activities Yokosuka, Yokosuka, Japan (Table 2). At LRRI, we performed viral isolation in A549 cell cultures, purification of HAdV genomic DNA, and molecular typing by restriction enzyme analysis or by PCR and Sanger sequencing of hexon and fiber genes as previously described $(8,16)$. At Walter Reed Army Institute of Research (Silver Spring, MD, USA), viral DNA samples received from LRRI were subjected to next-generation sequencing (NGS) fragment library preparation using QIAseq FX DNA Library Kit (QIAGEN, https://www.qiagen.com), followed by sequencing by using MiSeq Reagent Kit version 3 (600-cycle) and MiSeq sequencer (Illumina, https://www.illumina.com) (26). At the Pacific Rim Surveillance Hub (PRSH) of NHRC-OID, respiratory samples collected from persons meeting case definition for febrile respiratory illness were tested on the FilmArray Respiratory Pathogen Panel (Biofire Diagnostics, https://www.biofiredx.com), a multiplex panel consisting of 21 respiratory viral and bacterial pathogens. Adenovirus-positive samples were submitted to OID from Brian Allgood Army Community Hospital (BAACH), Seoul, South Korea. All samples from PRSH and BAACH sent to OID underwent further characterization that included additional typing by PCR amplification and sequencing of hypervariable region 7 of the hexon gene HVR7 as previously described (27). Clinical samples that failed to sequence were reflex-tested on type-specific assays that targeted the hexon gene, and were also inoculated in A549 cells to attempt viral isolation. We subjected all confirmed HAdV isolates to whole-genome NGS using Illumina Nextera XT library preparation kit and MiSeq System.

\section{Whole-Genome Sequence Assembly, Annotation, and Comparison}

We first analyzed NGS data acquired at Walter Reed Army Institute of Research with an in-house de novo pathogen discovery pipeline to identify possible 
Human Adenovirus Type 55

Table 2. HAdV type 55 strains used for genomic analysis for study of vius distribution, regional persistence, and genetic variability*

\begin{tabular}{|c|c|c|c|c|c|}
\hline Strain & Collection year & Collection site & Country & Source & $\begin{array}{c}\text { GenBank } \\
\text { accession no. }\end{array}$ \\
\hline HAdV-11/14 strain 273 & 1969 & Military camp & Spain & LRRI§ & MN654395 \\
\hline CADOH VRDL 76-0669 & 1976 & California & USA & LRRI & MN654394 \\
\hline CDC 97026382 & 1997 & South Dakota & USA & LRRI & MN654392 \\
\hline NAMRU3-E3 & 2000 & Alexandria & Egypt & LRRI & MN654380 \\
\hline NAMRU3-E4 & 2000 & Alexandria & Egypt & LRRI & MN654381 \\
\hline NAMRU3-E6 & 2000 & Alexandria & Egypt & LRRI & MN654385 \\
\hline NAMRU3-E66 & 2002 & Alexandria & Egypt & LRRI & MN654382 \\
\hline NAMRU3-E72 & 2000 & Alexandria & Egypt & LRRI & MN654383 \\
\hline NAMRU3 2005-909685 & 2005 & Cairo & Egypt & LRRI & MN654390 \\
\hline NAMRU3 2005-908017 & 2005 & Cairo & Egypt & LRRI & MN654391 \\
\hline NAMRU3 2007-905716 & 2007 & Cairo & Egypt & LRRI & MN654386 \\
\hline NAMRU3 2008-905223 & 2008 & Cairo & Egypt & LRRI & MN654384 \\
\hline NAMRU3 2009-908968 & 2009 & Cairo & Egypt & LRRI & MN654387 \\
\hline SNG1218 & 2005 & Singapore & Singapore & LRRI & MN654388 \\
\hline SNG1223 & 2005 & Singapore & Singapore & LRRI & MN654389 \\
\hline WPAFB24 & 2009 & $\mathrm{BAACH}$ & South Korea & LRRI & MN654379 \\
\hline WPAFB25 & 2009 & $\mathrm{BAACH}$ & South Korea & LRRI & MN654378 \\
\hline WPAFB48 & 2009 & $\mathrm{BAACH}$ & South Korea & LRRI & MN654377 \\
\hline WPAFB69 & 2009 & $\mathrm{BAACH}$ & South Korea & LRRI & MN654375 \\
\hline WPAFB75 & 2009 & BAACH & South Korea & LRRI & MN654376 \\
\hline WPAFB415 & 2012 & Misawa AB $\ddagger$ & Japan & LRRI & MN654393 \\
\hline NHRC557006 & 2017 & CFA Yokosukaף & Japan & NHRC & NA\# \\
\hline NHRC isolates, $n=50$ & 2017,2018 & BAACH & South Korea & NHRC & NA\# \\
\hline \multicolumn{6}{|c|}{$\begin{array}{l}\text { *An additional } 27 \text { full genome sequences from GenBank included in the analysis are listed in the Appendix Table 1. HAdV, human adenovirus. } \\
\text { †Brian Allgood Army Community Hospital at US Army Garrison Yongsan, Seoul, South Korea. } \\
\text { †Misawa Air Base, Misawa, Aomori, Japan. } \\
\text { §LRRI archival collection of isolates generated through collaborations with NHRC, United States Air Force School of Aerospace Medicine, US Naval } \\
\text { Medical Research Unit No. 3, US Centers for Disease Control and Prevention, and California Department of Health. } \\
\text { TCommander US Fleet Activities Yokosuka, Yokosuka, Japan. } \\
\text { \#lsolates were fully genome sequenced and found to be identical to the South Korea HAdV-55 strains. }\end{array}$} \\
\hline
\end{tabular}

mix of viruses (28). We assembled full genome sequences using NGS_Mapper (https://github.com/ UDBWRAIR/ngs_mapper) an in-house reference mapping pipeline built on BWA-MEM assembler $(\mathrm{H}$. Li, unpub. data, https://arxiv.org/abs/1303.3997v2). We used Geneious R10 (Biomatters Ltd., https:// www.geneious.com) and Integrative Genomics Viewer (IGV) software (Broad Institute, https://igv. org) for manual curation of the assembly, sequence alignment, annotation of genes, etc. We used the complete genome sequence of HAdV-55 strain QS-DLL/ China/ 2006 (GenBank accession no. FJ643676) $(29,30)$ as reference in reference mapping, annotation, and sequence comparison. We analyzed NGS data acquired at NHRC using the EDGE (Empowering the Development of Genomics Expertise) Bioinformatics Platform (https://edge.readthedocs.io) (31) and Lasergene software suite (DNASTAR, Inc., https://www.dnastar.com), and performed sequence alignment using BLAST (http:/ / blast.ncbi.nlm.nih.gov/Blast.cgi).

\section{Phylogenetic and Proteomic Analysis}

We used MAFFT and MEGA7 (32) to align HAdV55 genomic sequences. We determined general time reversible gamma-distributed invariant models of evolution using jModelTest2 (https://github.com/ ddarriba/jmodeltest2), and inferred a maximum likelihood phylogenetic tree using PhyML in MEGA7 (http:/ / www.megasoftware.net), with subtree pruning and regrafting and nearest-neighbor interchange tree search and Shimodiara-Hasegawa approximate likelihood ratio test for node confidence values.

We input the nucleotide sequence alignment and the GenBank feature table for the reference strain QS-DLL/China/2006 (FJ643676) into an in-house pipeline that annotates the sequences. To aid in visualization and comparison, we concatenated and aligned the annotated protein sequences using MUSCLE (http://www.drive5.com/muscle) in Geneious R10. We removed redundant identical sequences for further analysis. We visualized mismatches with an augmented version of the output using Highlighter software (https://www.hiv.lanl.gov/content/sequence/HIGHLIGHT/highlighter_top.html) (33).

\section{Results}

HAdV55 has been detected worldwide for decades (Table 1). We obtained complete genome sequences for a set of diverse HAdV-55 strains originating in 6 countries over many years and deposited them in GenBank under accession nos. MN654375-MN654395 (Table 2). The characterized strains include strain 273/ Spain/1969, originally identified as an intermediate variant 11/14 and isolated during an outbreak of ARD 
in the Spanish military (34); it is the earliest available HAdV-55 isolate and is therefore considered the prototype strain. In addition, the examined collection includes 2 strains isolated from civilians in the United States, 76-0669/USA/CA/1976 and 97026382/USA/ $\mathrm{SD} / 1997$, the strain isolated during a large ARD outbreak in a civilian job training facility in South Dakota (35); 10 HAdV-55 strains from 2 major cities in Egypt; 2 strains from a Singapore military base; 2 HAdV-55 strains isolated in different locations and years in Japan; and 50 recent strains from South Korea.

The genome sequences of HAdV-55 strains are highly similar, with 132 or fewer nucleotide differences out of the $34.8 \mathrm{~kb}$ genome (i.e., genomic nucleotide divergence $\leq 0.38 \%$ among all the strains). The inferred phylogenetic tree of HAdV-55 lineage shows the examined strains mostly clustered together by collection country (South Korea, China, Singapore, and Egypt), rather than collection year (Figure 1). The results demonstrate the long-term regional persistence of HAdV-55 infection, which appears to span for years. The 3 isolates collected in 2005 from Singapore were identical to each other and located within the clade of Egypt isolates from 2000-2009. We detected $<9$ nt differences among all 14 Egypt and Singapore isolates examined (12 from this study; 2 from GenBank). The genome sequences of isolate NHRC557006/Japan/2017 and all the South Korea isolates from this study and in GenBank were found to be identical, except for the differences in the length of the noncoding poly(A) or poly(T) sequences. A previous isolate, WPAFB415/ Japan/2012, was phylogenetically distinct from isolate NHRC557006/Japan/2017 and South Korea strains, with proximity with the Egypt strains. Likewise, isolates from South Korea clustered in a monophyletic clade located proximate to the clade of China strains. Another isolate from China was found outside of the Chinese clade and was more related to a strain from Argentina. Nevertheless, we show a highly localized persistence of HAdV-55. GenBank had 1 available genome sequence for an HAdV-55 strain from Argentina, ak36_AdV11a/ARG/2005 (accession no. JX423384); however, Kajon et al. (16) performed thorough restriction enzyme analysis characterization and compared hexon and fiber gene sequences for 7 Argentina HAdV-55 isolates collected in 2000-2005. Their results suggest these are isolates of a single strain. Of interest, we found the virtual restriction profiles with BamHI, BclI, BglII, BstEII, HindIII, HpaI, PstI, SmaI, and XbaI derived from genome sequence JX423384 to be $100 \%$ identical to the patterns reported for HAdV-55 strains by Kajon et al., suggesting a single strain was responsible for multiple ARD cases in Argentina.
We compared amino acid sequences of all open reading frames (ORF) among HAdV-55 strains (Figure 2; Appendix Table, https://wwwnc.cdc.gov/EID/ article/26/7/19-1707-App1.xlsx) and found very few amino acid differences. Out of 10,925 aa in the annotated 37 proteins, only $2-15$ aa residues in each strain are different from consensus protein sequences (amino acid variation $\leq 0.14 \%$ ) among the HAdV-55 strains. No single annotated protein had $>2$ amino acid differences compared with the consensus except BJ01/CHN/2011 (GenBank accession no. JX491639), which had 6 amino acid substitutions in the 246 aa L3 pVI $26.6 \mathrm{kDa}$ protein and 3 amino acid mutations in the 812 aa L4 100K protein (Appendix Table). Seven proteins, L2 pVII $21.3 \mathrm{kDa}$ protein, L3 pVI $26.6 \mathrm{kDa}$ protein, L3 23K $23.7 \mathrm{kDa}$ protein, E2A $58.3 \mathrm{kDa}$ DNA binding protein, L4 pVIII $25 \mathrm{kDa}$ protein, E4 ORF3 13.6 $\mathrm{kDa}$ protein, and E4 ORF2 14.3 kDa protein, were identical among all the examined strains.

Our analyses showed that hexon, fiber, and penton base proteins do not have more amino acid residue changes when compared with other proteins (Figure 2). We detected a few insertions and deletions in intergenic untranslated regions in several genomes. The strain USA/1976 has 2 deletions (of 3 aa each) in the coding sequences for L4 100K/91 kDa protein and L4 $22 \mathrm{~K} / 21.6 \mathrm{kDa}$ protein (Appendix Table). It is remarkable that there were no amino acid differences among South Korea isolates from 2009-2018. Despite the very few amino acid substitutions, most residue changes resulted in amino acids of different chemical structures, which may potentially affect protein functionality (Appendix Table). Several amino acid mutations were strain-specific and not seen in other strains or other related HAdV types. One specific example is that the P18S mutation in the terminal protein precursor (pTP) was only found in the South Korea strain. This position was highly conserved (a proline) in all other examined adenoviruses including HAdVs of species B and related simian or gorilla adenoviruses (GenBank accession nos. AP_000267, AP_000305, YP_006272955, ADQ38372). The strain BJ01/CHN/2011 was phylogenetically more closely related to the South Korea strain and had the same pTP sequence with the P18S mutation.

We obtained a total of 51 HAdV-55 isolates, 50 recovered from US military active duty personnel in South Korea and 1 from a US military dependent in Japan, through PRSH efforts. Whole-genome sequencing and sequence data analysis confirmed all strains to be identical to the South Korea strain in full agreement with the recent reports on HAdV-55 circulation in South Korea and the high number of ARD cases documented among the South Korea military $(18,36)$. 


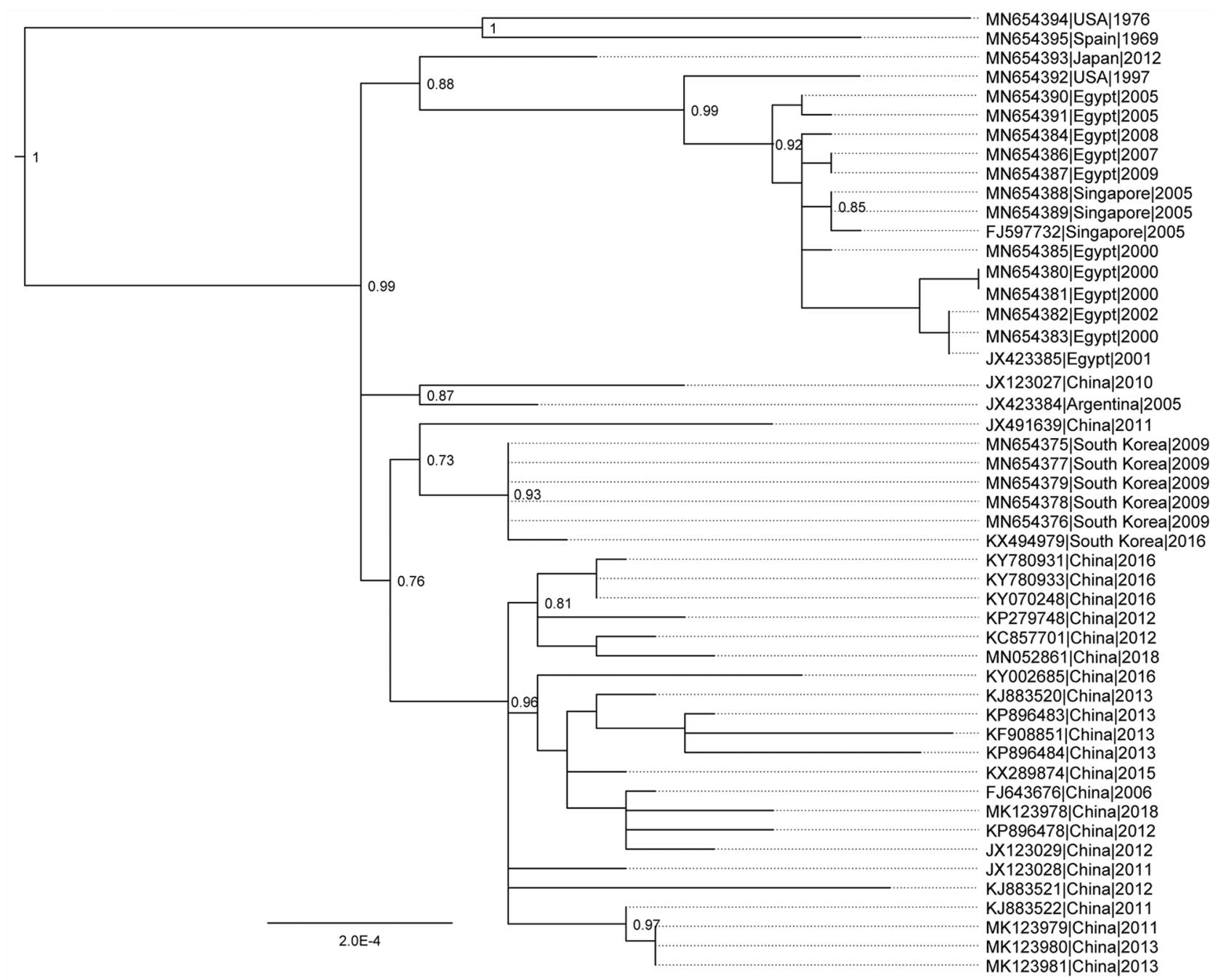

Figure 1. Phylogeny of HAdV-55 based on whole-genome sequences for study of virus distribution, regional persistence, and genetic variability. The phylogenetic tree was generated using the maximum-likelihood method with subtree pruning and regrafting and nearestneighbor interchange tree search and the Shimodiara-Hasegawa approximate likelihood ratio test for node confidence values. Node confidence values were estimated using approximate likelihood ratio test and the tree was rooted on a HAdV-14 clade as an outgroup (not shown). GenBank accession numbers for isolates are provided. Scale bar indicates node confidence value. HAdV, human adenovirus.

\section{Discussion}

Recent reports describing large numbers of HAdV55-associated ARD cases in both China and South Korea, including outbreaks and some deaths in both civilian and military communities, have raised concerns about the possibility of global transmission events, similar to those described for severe acute respiratory syndrome or Middle East respiratory syndrome (21,37-43). Outbreaks and isolated cases of HAdV-55-associated ARD have been reported in the literature in other countries, such as Turkey (44), Israel (45), and France (46) since 2005. Possible reasons for underdetection and underreporting of HAdV-55 are its recent designation in 2013 as a discrete adenovirus type, following its recognition as an intertypic recombinant (P14H11F14) (17), and probably also molecular typing practices based solely on partial sequencing of the hexon gene. Molecular diagnosis of HAdV-55 and other intertypic recombinant HAdV genotypes requires a PCR-based assay targeting $\geq 2$ regions of the genome, the penton base and hexon or the hexon and fiber genes; such assays would greatly improve molecular surveillance practices. The prototype strain, 273/Spain/1969, was detected in association with a severe ARD outbreak involving military recruits in Spain and reported as a serologically intermediate variant 11/14 (34). The US strain 97026382/South Dakota/1997, originally reported as HAdV-11 (35) and subsequently described as genome type 11a (16), caused a large ARD outbreak in a job 
training center. It is notable that the circulation of these viruses was not detected or reported in Europe or North America in the years following the detection of either the prototype or US strain. On the other hand, the long persistence of HAdV-55 in China and South Korea suggests continuous transmission and endemicity. If strains circulating in China or South Korea can cause repeated outbreaks, that indicates an important change in the epidemiologic pattern of HAdV-55 infection: from sporadic epidemic outbreak to persistent endemicity. The identification of factors that affect HAdV-55 transmission, from novel functional changes in the viruses to social or environmental changes, warrants further investigation.

Our study and others have shown that recent HAdV-55 genomes share a nucleotide identity $>99.7 \%$ $(22,23,29)$. The nucleotide variations are located all over the genome and are not concentrated in any particular genomic regions. We noted amino acid substitutions on proteins essential for viral replication, such as L4 100K, which may affect virus growth phenotype, antigenicity, infectivity, or virulence. Terminal protein precursor (pTP) and its proteaseprocessed derivatives, intermediate terminal protein (iTP) and mature terminal protein (TP), play crucial and complex roles in adenovirus genome replication and virus maturation. The mutation P18S is unique to South Korea strains and located in a conserved region of pTP. Because the structure of pTP protein of adenovirus has not been resolved, it is unknown whether the P to $S$ mutation will affect pTP structure and consequently change protease cleavage pattern of pTP, DNA replication, and genome packaging. Earlier work by Hay et al. $(47,48)$ demonstrated that point mutations and deletions generated on pTP affect DNA replication activity in vitro. Flint et al. (49) showed that G315V substitution in pTP of HAdV-5 impaired pTP maturation leading to reduced infectivity. The remaining genomic changes of HAdV-55 appear to be largely trivial and not likely responsible

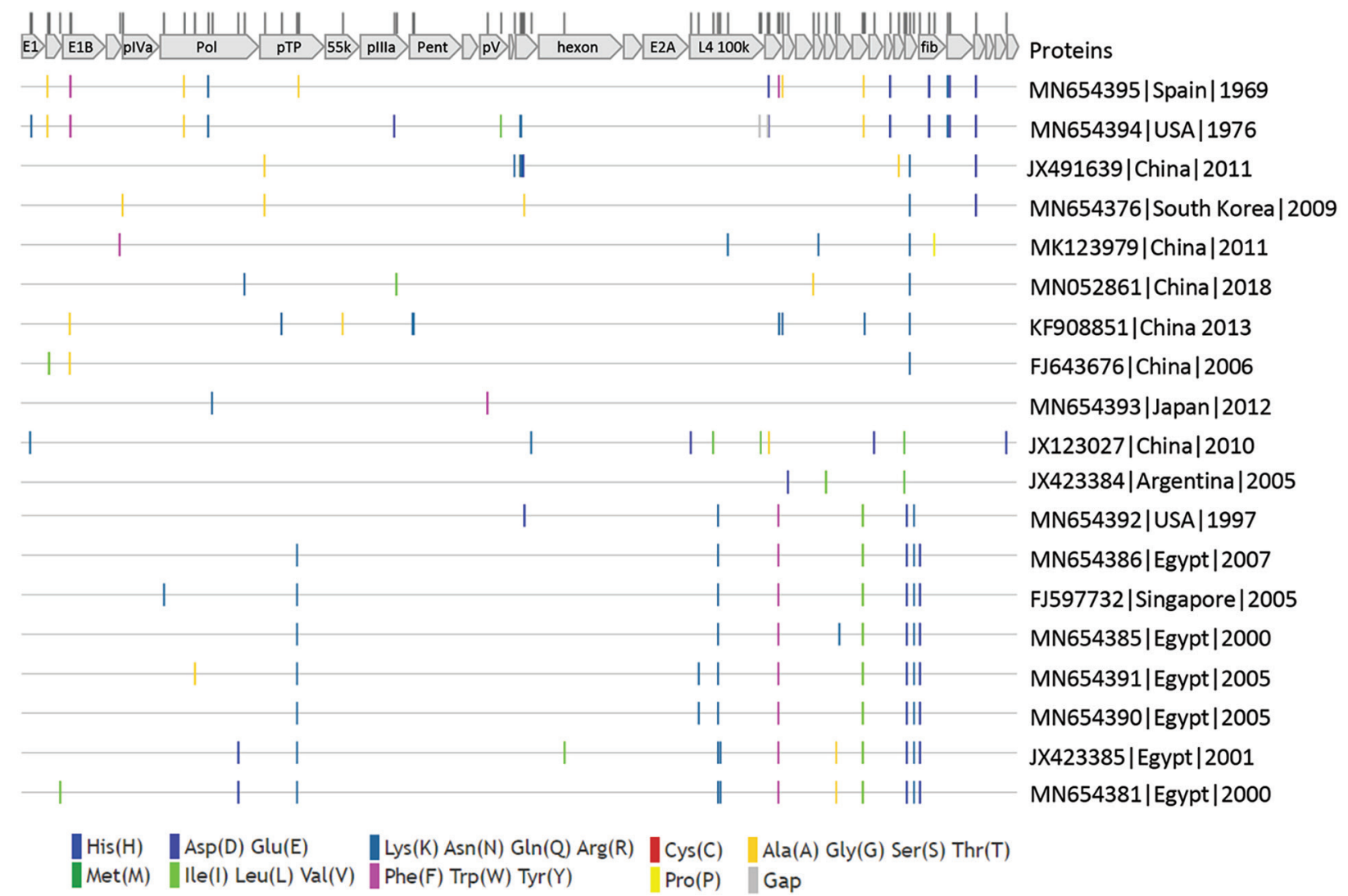

Figure 2. Protein sequence variations among human adenovirus type 55 strains for study of virus distribution, regional persistence, and genetic variability. Protein sequences were concatenated and aligned. Amino acid differences compared with the consensus were visualized with Highlighter software (https://www.hiv.lanl.gov/content/sequence/HIGHLIGHT/highlighter_top.html). Redundant sequences are not shown. The locations of proteins and variant amino acid residues are shown at top. GenBank accession numbers for isolates are provided. Ala, alanine; Arg, arginine; Asn, asparagine; Asp, aspartate; Cys, cysteine; Gln, glutamine; Glu, glutamate; Gly, glycine; His, histidine; Ile, isoleucine; Leu, leucine; Lys, lysine; Met, methionine; Phe, phenylalanine, Pro, proline; Ser, serine; Thr, threonine; Trp, tryptophan; Tyr, tyrosine; Val, valine. 
for the increased incidence of outbreaks and disease severity. Further investigation and comparison of these strains on virology, molecular biology, and biochemistry perspectives will provide solid evidence to clarify whether some of the current strains are more infectious or virulent and therefore pose higher risks to human health. Detailed molecular epidemiology study, such as reported by Jing et al. on household HAdV-55 transmission (50), is warranted to enhance etiologic understanding of HAdV-55-caused ARD for accurate and timely diagnosis and disease prevention.

The United States has the highest number of domestic and international trade and travel visits in the world and is therefore highly susceptible to importation and dispersion of incoming pathogens such as HAdV-55. Indeed, isolated HAdV-55 cases and at least one outbreak have occurred in the United States with no clear identification of the source. Of interest, as suggested by the results of our phylogenetic analysis, the 2 US strains were not apparently related to each other. Therefore, implementation of enhanced surveillance, including typing of clinically relevant HAdV strains, is needed, along with proper design of countermeasures such as rapid diagnostics, treatments, and novel vaccines.

A limitation of this study is the lack of detailed clinical data and travel history. More information is needed for accurate risk assessment of disease transmission. It is unclear whether NHRC557006/ Japan/2017 was introduced from South Korea by travel, or whether a cryptic circulation of the South Korea strain in Japan has yet to be detected. Similarly, without reports on additional HAdV-55 cases in Japan, it remains unknown which HAdV-55 strain, the South Korea strain, WPAFB415/Japan/2012, or other unknown strains, are circulating in Japan. A large number of HAdV-55-associated ARD cases were detected in both South Korea military personnel and US military personnel stationed in South Korea. Determining whether infected persons trained together or participated in same military events, how long and how often they were in close contact, the timeline of infection, and whether ARD outbreaks during an extended training period were underreported is important. It is worth noting that US active duty military in South Korea were vaccinated against HAdV-4 and HAdV-7, which suggests that the HAdV-4 and HAdV-7 vaccine formulation does not confer adequate protection against HAdV55. Our ongoing and planned studies on HAdV-55specific serologic surveys before, during, and after military deployment, as well as local serologic surveys in South Korea and Japan, will contribute to comprehensive understanding of HAdV-55 prevalence and enable data-driven decisions on the necessity of enhanced surveillance and development of effective prophylaxes.

\section{Acknowledgment}

We thank research collaborators for generous sharing of specimens which made this study possible. We also thank James S. Hilaire, Nicole R. Nicholas, Tuan K. Nguyen, and April N. Griggs for their assistance in project management and sample tracking, storage, and retrieval. Finally, we thank the Defense Health Agency Immunization Healthcare Division (DHA-IHD), Global Emerging Infections Surveillance and Response System (GEIS), Division of the Armed Forces Health Surveillance Branch.

\section{About the Author}

Dr. Hang is a molecular microbiologist at Walter Reed Army Institute of Research, Silver Spring, Maryland, USA. His primary research interest is pathogen discovery in clinical and environmental specimens.

\section{References}

1. Sanchez JL, Binn LN, Innis BL, Reynolds RD, Lee T, Mitchell-Raymundo F, et al. Epidemic of adenovirus-induced respiratory illness among US military recruits: epidemiologic and immunologic risk factors in healthy, young adults. J Med Virol. 2001;65:710-8. https:/ / doi.org/10.1002/jmv.2095

2. Kolavic-Gray SA, Binn LN, Sanchez JL, Cersovsky SB, Polyak CS, Mitchell-Raymundo F, et al. Large epidemic of adenovirus type 4 infection among military trainees: epidemiological, clinical, and laboratory studies. Clin Infect Dis. 2002;35:808-18. https:/ / doi.org/10.1086/342573

3. Padin DS, Faix D, Brodine S, Lemus H, Hawksworth A, Putnam S, et al. Retrospective analysis of demographic and clinical factors associated with etiology of febrile respiratory illness among US military basic trainees. BMC Infect Dis. 2014;14:576. https:// doi.org/10.1186/s12879-014-0576-2

4. Kajon AE, Lamson DM, St George K. Emergence and re-emergence of respiratory adenoviruses in the United States. Curr Opin Virol. 2019;34:63-9. https:/ / doi.org/ 10.1016/j.coviro.2018.12.004

5. Gray GC, Goswami PR, Malasig MD, Hawksworth AW, Trump DH, Ryan MA, et al.; Adenovirus Surveillance Group. Adult adenovirus infections: loss of orphaned vaccines precipitates military respiratory disease epidemics. Clin Infect Dis. 2000;31:663-70. https://doi.org/10.1086/313999

6. Blasiole DA, Metzgar D, Daum LT, Ryan MA, Wu J, Wills C, et al. Molecular analysis of adenovirus isolates from vaccinated and unvaccinated young adults. J Clin Microbiol. 2004;42:1686-93. https:/ / doi.org/10.1128/JCM.42.4.16861693.2004

7. Kuschner RA, Russell KL, Abuja M, Bauer KM, Faix DJ, Hait H, et al.; Adenovirus Vaccine Efficacy Trial Consortium. A phase 3, randomized, double-blind, placebo-controlled study of the safety and efficacy of the live, oral adenovirus type 4 and type 7 vaccine, in U.S. military recruits. Vaccine. 2013;31:2963-71. https:/ / doi.org/10.1016/ j.vaccine.2013.04.035 
8. Kajon AE, Hang J, Hawksworth A, Metzgar D, Hage E, Hansen CJ, et al. Molecular epidemiology of adenovirus type 21 respiratory strains isolated from US military trainees (1996-2014). J Infect Dis. 2015;212:871-80. https:/ / doi.org/ 10.1093/infdis/jiv141

9. Sanchez JL, Cooper MJ, Myers CA, Cummings JF, Vest KG, Russell KL, et al. Respiratory infections in the U.S. military: recent experience and control. Clin Microbiol Rev. 2015;28:743-800. https:/ / doi.org/10.1128/CMR.00039-14

10. Clemmons NS, McCormic ZD, Gaydos JC, Hawksworth AW, Jordan NN. Acute respiratory disease in US Army trainees 3 years after reintroduction of adenovirus vaccine. Emerg Infect Dis. 2017;23:95-8. https:// doi.org/10.3201/eid2301.161297

11. Ismail AM, Cui T, Dommaraju K, Singh G, Dehghan S, Seto J, et al. Genomic analysis of a large set of currently-and historically-important human adenovirus pathogens. Emerg Microbes Infect. 2018;7:10. https:/ / doi.org/10.1038/s41426017-0004-y

12. Dhingra A, Hage E, Ganzenmueller T, Böttcher S, Hofmann J, Hamprecht K, et al. Molecular evolution of human adenovirus (HAdV) species C. Sci Rep. 2019;9:1039. https:/ / doi.org/10.1038/s41598-018-37249-4

13. Ismail AM, Lee JS, Lee JY, Singh G, Dyer DW, Seto D, et al. Adenoviromics: mining the human adenovirus species D genome. Front Microbiol. 2018;9:2178. https:/ / doi.org/ 10.3389/fmicb.2018.02178

14. Li QG, Hambraeus J, Wadell G. Genetic relationship between thirteen genome types of adenovirus 11,34, and 35 with different tropisms. Intervirology. 1991;32:338-50. https://doi.org/10.1159/000150218

15. Hierholzer JC, Pumarola A. Antigenic characterization of intermediate adenovirus 14-11 strains associated with upper respiratory illness in a military camp. Infect Immun. 1976;13:354-9. https:// doi.org/10.1128/IAI.13.2.354-359.1976

16. Kajon AE, de Jong JC, Dickson LM, Arron G, Murtagh P, Viale D, et al. Molecular and serological characterization of species B2 adenovirus strains isolated from children hospitalized with acute respiratory disease in Buenos Aires, Argentina. J Clin Virol. 2013;58:4-10. https:/ / doi.org/ 10.1016/j.jcv.2013.06.030

17. Seto D, Jones MS, Dyer DW, Chodosh J. Characterizing, typing, and naming human adenovirus type 55 in the era of whole genome data. J Clin Virol. 2013;58:741-2. https:// doi.org/10.1016/j.jcv.2013.09.025

18. Ko JH, Woo HT, Oh HS, Moon SM, Choi JY, Lim JU, et al. Ongoing outbreak of human adenovirus-associated acute respiratory illness in the Republic of Korea military, 2013 to 2018. Korean J Intern Med (Korean Assoc Intern Med). 2019;kjim.2019.092.

19. Gao HW, Wei MT, Fan HJ, Ding H, Wei W, Liu ZQ, et al. Dynamic changes in clinical characteristics during an outbreak of human adenovirus serotype 55 in China. Disaster Med Public Health Prep. 2018;12:464-9. https://doi.org/10.1017/dmp.2015.185

20. Zheng X, Rong X, Feng Y, Sun X, Li L, Wang Q, et al. Seroprevalence of neutralizing antibodies against adenovirus type 14 and 55 in healthy adults in Southern China. Emerg Microbes Infect. 2017;6:e43. https:// doi.org/10.1038/emi.2017.29

21. Lu QB, Tong YG, Wo Y, Wang HY, Liu EM, Gray GC, et al. Epidemiology of human adenovirus and molecular characterization of human adenovirus 55 in China, 2009-2012. Influenza Other Respir Viruses. 2014;8:302-8. https:/ / doi.org/10.1111/irv.12232

22. Cheng Z, Yan Y, Jing S, Li WG, Chen WW, Zhang J, et al. Comparative genomic analysis of re-emergent human adenovirus type 55 pathogens associated with adult severe community-acquired pneumonia reveals conserved genomes and capsid proteins. Front Microbiol. 2018;9:1180. https:// doi.org/10.3389/fmicb.2018.01180

23. Wang W, Liu Y, Zhou Y, Gu L, Zhang L, Zhang X, et al. Whole-genome analyses of human adenovirus type 55 emerged in Tibet, Sichuan and Yunnan in China, in 2016. PLoS One. 2017;12:e0189625. https:/ / doi.org/10.1371/ journal.pone.0189625

24. Gu SH, Song DH, Lee D, Huh K, Yoo H, Oh HS, et al. Complete genome sequence of human adenovirus type 55 associated with acute respiratory disease, isolated from a military base in the Republic of Korea. Genome Announc. 2017;5:5. https://doi.org/10.1128/genomeA.01565-16

25. Zhang Q, Seto D, Cao B, Zhao S, Wan C. Genome sequence of human adenovirus type 55 , a re-emergent acute respiratory disease pathogen in China. J Virol. 2012;86:124412. https:// doi.org/10.1128/JVI.02225-12

26. Hang J, Vento TJ, Norby EA, Jarman RG, Keiser PB, Kuschner RA, et al. Adenovirus type 4 respiratory infections with a concurrent outbreak of coxsackievirus A21 among United States Army Basic Trainees, a retrospective viral etiology study using next-generation sequencing. J Med Virol. 2017;89:1387-94. https:/ / doi.org/10.1002/jmv.24792

27. Sarantis H, Johnson G, Brown M, Petric M, Tellier R. Comprehensive detection and serotyping of human adenoviruses by PCR and sequencing. J Clin Microbiol. 2004; 42:3963-9. https:// doi.org/10.1128/JCM.42.9.3963-3969.2004

28. Kilianski A, Carcel P, Yao S, Roth P, Schulte J, Donarum GB, et al. Pathosphere.org: pathogen detection and characterization through a web-based, open source informatics platform. BMC Bioinformatics. 2015;16:416. https://doi.org/10.1186/s12859-015-0840-5

29. Yang Z, Zhu Z, Tang L, Wang L, Tan X, Yu P, et al. Genomic analyses of recombinant adenovirus type 11a in China. J Clin Microbiol. 2009;47:3082-90. https:/ / doi.org/ 10.1128/JCM.00282-09

30. Walsh MP, Seto J, Jones MS, Chodosh J, Xu W, Seto D. Computational analysis identifies human adenovirus type 55 as a re-emergent acute respiratory disease pathogen. J Clin Microbiol. 2010;48:991-3. https:/ / doi.org/10.1128/JCM.01694-09

31. Li PE, Lo CC, Anderson JJ, Davenport KW, Bishop-Lilly KA, $\mathrm{Xu} \mathrm{Y}$, et al. Enabling the democratization of the genomics revolution with a fully integrated web-based bioinformatics platform. Nucleic Acids Res. 2017;45:67-80. https://doi.org/10.1093/nar/gkw1027

32. Kumar S, Stecher G, Tamura K. MEGA7: Molecular Evolutionary Genetics Analysis version 7.0 for bigger datasets. Mol Biol Evol. 2016;33:1870-4. https:/ / doi.org/ 10.1093/molbev/msw054

33. Keele BF, Giorgi EE, Salazar-Gonzalez JF, Decker JM, Pham KT, Salazar MG, et al. Identification and characterization of transmitted and early founder virus envelopes in primary HIV-1 infection. Proc Natl Acad Sci U S A. 2008;105:7552-7. https://doi.org/10.1073/pnas.0802203105

34. Hierholzer JC, Pumarola A, Rodriguez-Torres A, Beltran M. Occurrence of respiratory illness due to an atypical strain of adenovirus type 11 during a large outbreak in Spanish military recruits. Am J Epidemiol. 1974;99:434-42. https:/ / doi.org/10.1093/oxfordjournals.aje.a121632

35. Centers for Disease Control and Prevention (CDC). Civilian outbreak of adenovirus acute respiratory disease-South Dakota, 1997. MMWR Morb Mortal Wkly Rep. 1998;47:567-70.

36. Yoo H, Gu SH, Jung J, Song DH, Yoon C, Hong DJ, et al. Febrile respiratory illness associated with human adenovirus type 55 in South Korea military, 2014-2016. Emerg Infect Dis. 2017;23:1016-20. https://doi.org/10.3201/eid2306.161848 
37. Heo JY, Noh JY, Jeong HW, Choe KW, Song JY, Kim WJ, et al. Molecular epidemiology of human adenovirusassociated febrile respiratory illness in soldiers, South Korea. Emerg Infect Dis. 2018;24:1221-7. https:/ / doi.org/10.3201/ eid2407.171222

38. Yoon H, Jhun BW, Kim H, Yoo H, Park SB. Characteristics of adenovirus pneumonia in Korean military personnel, 2012-2016. J Korean Med Sci. 2017;32:287-95. https://doi.org/10.3346/jkms.2017.32.2.287

39. Park JY, Kim BJ, Lee EJ, Park KS, Park HS, Jung SS, et al. Clinical features and courses of adenovirus pneumonia in healthy young adults during an outbreak among Korean military personnel. PLoS One. 2017;12:e0170592. https://doi.org/10.1371/journal.pone.0170592

40. Dongliu Y, Guoliang Y, Haocheng X, Shuaijia Q, Li B, Yanglei J. Outbreak of acute febrile respiratory illness caused by human adenovirus B P14H11F14 in a military training camp in Shandong China. Arch Virol. 2016;161:2481-9. https://doi.org/10.1007/s00705-016-2949-x

41. Li X, Kong M, Su X, Zou M, Guo L, Dong X, et al. An outbreak of acute respiratory disease in China caused by human adenovirus type B55 in a physical training facility. Int J Infect Dis. 2014;28:117-22. https:/ / doi.org/10.1016/ j.ijid.2014.06.019

42. Gu L, Qu J, Sun B, Yu X, Li H, Cao B. Sustained viremia and high viral load in respiratory tract secretions are predictors for death in immunocompetent adults with adenovirus pneumonia. PLoS One. 2016;11:e0160777. https://doi.org/ 10.1371/journal.pone.0160777

43. Zhang SY, Luo YP, Huang DD, Fan H, Lu QB, Wo Y, et al. Fatal pneumonia cases caused by human adenovirus 55 in immunocompetent adults. Infect Dis (Lond). 2016;48:40-7. https://doi.org/10.3109/23744235.2015.1055585

44. Chmielewicz B, Benzler J, Pauli G, Krause G, Bergmann F, Schweiger B. Respiratory disease caused by a species B2 adenovirus in a military camp in Turkey. J Med Virol. 2005;77:232-7. https://doi.org/10.1002/jmv.20441

45. Salama M, Amitai Z, Amir N, Gottesman-Yekutieli T, Sherbany H, Drori Y, et al. Outbreak of adenovirus type 55 infection in Israel. J Clin Virol. 2016;78:31-5. https:/ / doi.org/ 10.1016/j.jcv.2016.03.002

46. Lafolie J, Mirand A, Salmona M, Lautrette A, Archimbaud C, Brebion A, et al. Severe pneumonia associated with adenovirus type 55 infection, France, 2014. Emerg Infect Dis. 2016;22:2012-4. https:/ / doi.org/10.3201/eid2211.160728

47. Botting $\mathrm{CH}$, Hay RT. Role of conserved residues in the activity of adenovirus preterminal protein. J Gen Virol. 2001;82:1917-27. https:/ / doi.org/10.1099/0022-1317-82-81917

48. Liu H, Naismith JH, Hay RT. Adenovirus DNA replication. Curr Top Microbiol Immunol. 2003;272:131-64. https://doi.org/10.1007/978-3-662-05597-7_5

49. Kato SE, Chahal JS, Flint SJ. Reduced infectivity of adenovirus type 5 particles and degradation of entering viral genomes associated with incomplete processing of the preterminal protein. J Virol. 2012;86:13554-65. https://doi.org/10.1128/JVI.02337-12

50. Jing S, Zhang J, Cao M, Liu M, Yan Y, Zhao S, et al. Household transmission of human adenovirus type 55 in case of fatal acute respiratory disease. Emerg Infect Dis. 2019;25:1756-8. https:// doi.org/10.3201/eid2509.181937

Address for correspondence: Jun Hang, Viral Diseases Branch, Walter Reed Army Institute of Research, Silver Spring, MD 20910, USA; email: jun.hang.civ@mail.mil

\section{EID Podcast:}

Visions of Matchstick Men and Icons of Industrialization

Byron Breedlove, managing editor of the journal, discusses and reads his November 2017 cover art essay. This cover (Going to Work, 1943) is by English artist Laurence Stephen Lowry (1887-1976) who died of pneumonia in 1976.

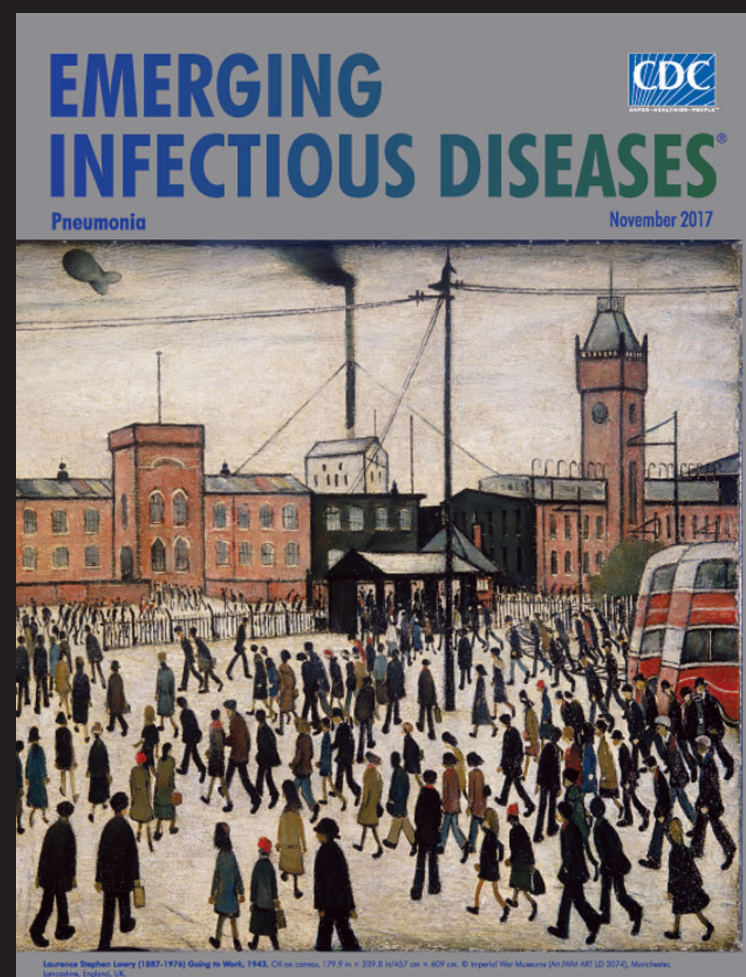

Visit our website to listen: https:/ / www2c.cdc.gov/ podcasts/player.asp?f=8647173 EMERGING
INFECTIOUS DISEASES 